\title{
Temperature-driven gapless topological insulator
}

\author{
Miguel Gonçalves, ${ }^{1}$ Pedro Ribeiro, ${ }^{1,2}$ Rubem Mondaini, ${ }^{2}$ and Eduardo V. Castro ${ }^{1,2,3}$ \\ ${ }^{1}$ CeFEMA, Instituto Superior Técnico, Universidade de Lisboa, Av. Rovisco Pais, 1049-001 Lisboa, Portugal \\ ${ }^{2}$ Beijing Computational Science Research Center, Beijing 100084, China \\ ${ }^{3}$ Centro de Física das Universidades do Minho e Porto, \\ Departamento de Física e Astronomia, Faculdade de Ciências, \\ Universidade do Porto, 4169-007 Porto, Portugal
}

\begin{abstract}
We investigate the phase diagram of the Haldane-Falicov-Kimball model - a model combining topology, interactions and spontaneous disorder at finite temperatures. Using an unbiased numerical method, we map out the phase diagram on the interaction-temperature plane. Along with known phases, we unveil an insulating charge ordered state with gapless excitations and a temperaturedriven gapless topological insulating phase. Intrinsic - temperature generated - disorder, is the key ingredient explaining the unexpected behavior. Our findings support the possibility of having temperature-driven topological phase transitions into gapped and gapless topological insulating phases in systems with a large mass unbalance in fermionic species.
\end{abstract}

Understanding the effects of disorder, interactions and temperature on topological phases of matter is essential to predict the topological properties and their stability in real-world materials [1]. Some of these effects are quite subtle and may have dichotomic features. For example, topological phases are suppressed in the presence of strong nearest-neighbor (NN) [2] or Hubbard-like interactions [3-12]. However, interaction-induced magnetic order was found to coexist with topological phases [13$16]$ and some studies showed that interactions themselves could induce a topological phase on a trivial band, forming the so-called topological Mott insulator [17-24]. Even if this phase is disputed outside the mean-field scope in some models [25-28], it has been confirmed in others $[29,30]$.

The influence of correlations at finite temperatures on topological insulators (TI) also shows opposite trends [31-33]. Although thermal fluctuations are responsible for the destruction of topological order when large enough [34,35], they can also drive different types of topological phases [31, 36].

The role of disorder on topological phases is also subtle. For TI within the unitary class [37-39] (for which time-reversal symmetry is broken), disorder effects localize every eigenstate except two bulk extended states that carry opposite Chern numbers [40, 41]. The merging of these states, for a sufficiently large disorder strength, is associated with the destruction of the topological phase. Interestingly, a disorder-induced transition into a new topologically nontrivial phase - the topological Anderson insulator (TAI) - was also shown to be possible [42-46].

In this Letter, we explore some of the dichotomic aspects above by fully characterizing an interacting quantum model that crucially combines non-trivial topology, disorder, temperature, and interaction effects, and which can be efficiently studied by unbiased numerical methods. Our main results are summarized in Fig. 1, which depicts the different phases as a function of the temperature, $T$, and of the interaction magnitude, $U$. As a central result, topological order was found to appear for intermediate $U$ values when $T$ is increased, and to extend into the gapless region of the phase diagram at higher $T$, giving rise to a temperature-driven gapless topological insulating phase (GTI).

Model. - Our starting point is the Falicov-Kimball model (FKM) [47], a limiting case of the Hubbard model for which one of the spin fermion species is infinitely massive, rendering these fermions - the $f$-electrons - immobile. For a half-filled bipartite lattice at $T=0$, the $f$ -

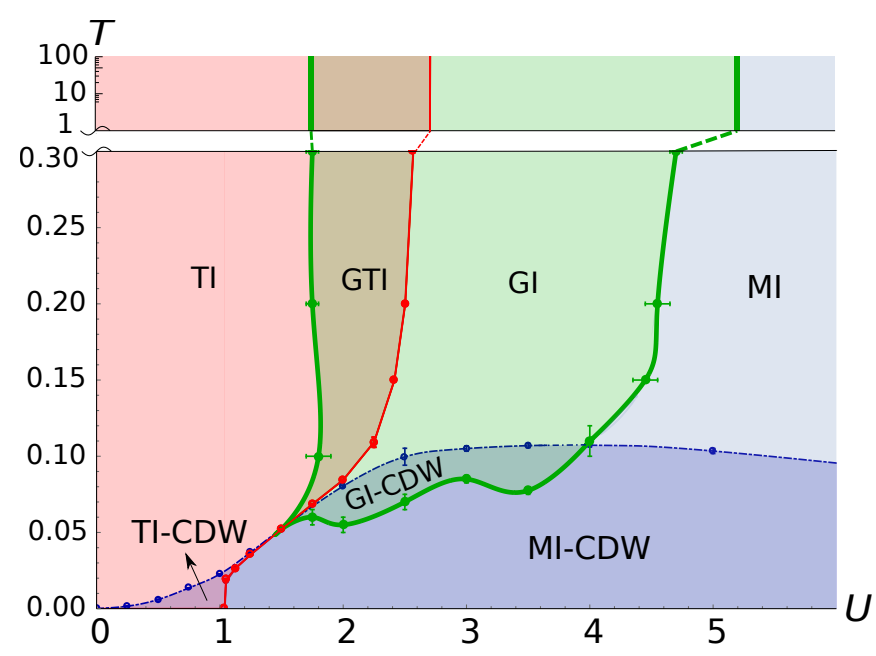

FIG. 1. Phase diagram of the HFKM in the interaction temperature plane obtained with the Monte Carlo method. Phases at intermediate- to high- $T$, outside the charge density wave phase (CDW): topological insulator (TI) for small $U$, gapless topological insulator (GTI) and gapless insulator (GI) for intermediary $U$, and Mott-like insulating phase (MI) for large $U$. Phases at low- $T$, inside the CDW phase: phases with similar features as their high- $T$ counterparts were found and the suffix "-CDW" was added. The thin (red) and dasheddotted (blue) curves correspond, respectively, to the topological and CDW phase transitions and the thick (green) curve bounds the gapless region of the phase diagram. 
electrons order in a charge density wave (CDW) state for any finite value of the interaction strength between the localized and itinerant electrons [48-51]. Recently, the full $T$-dependent phase diagram of the $2 \mathrm{D}$ FKM was obtained with Monte Carlo (MC) techniques unveiling an Anderson insulating phase overlooked in previous studies [52]. The averaging on the configurations of $f$-electrons, sampled according to the total partition function with the MC method, acts as a disorder potential to itinerant electrons rendering possible that their eigenstates become localized without the presence of explicit disorder. We combine the interacting nature of the FKM with the topological features of the first theoretical model of a TI under a zero net magnetic field - the Haldane model [53] - which, although robust to small disorder, has its topological properties destroyed for large enough disorder strengths [54-57].

The Hamiltonian of the Haldane-Falicov-Kimball model (HFKM) is defined as

$$
\begin{aligned}
\hat{H} & =-t \sum_{\langle i, j\rangle} \hat{c}_{i}^{\dagger} \hat{c}_{j}+\mathrm{i} t_{2} \sum_{\langle\langle i, j\rangle\rangle} \nu_{i j} \hat{c}_{i}^{\dagger} \hat{c}_{j}+\text { H.c. } \\
& +U \sum_{i} \hat{c}_{i}^{\dagger} \hat{c}_{i} n_{i}^{f}-\sum_{i}\left(\mu_{c} \hat{c}_{i}^{\dagger} \hat{c}_{i}+\mu_{f} n_{i}^{f}\right),
\end{aligned}
$$

depicting a species of itinerant electrons ( $c$-electrons) with creation operators $\hat{c}_{i}^{\dagger}$ and another of localized electrons ( $f$-electrons) whose local density at site $i$ is given by the number $n_{i}^{f}$. The operators $\hat{c}_{i}^{\dagger}=\hat{c}_{i, A}^{\dagger}, \hat{c}_{i, B}^{\dagger}$ are defined in the two interpenetrating triangular sublattices $A$ and $B$ that form the honeycomb lattice shown in the sketch in Fig. 2 a, with total volume $V=2 L^{2}$, where $L$ indicates the linear number of unit cells. The first term is the kinetic energy of the itinerant electrons associated with $\mathrm{NN}$ hoppings, with $t$ being the hopping integral for $\mathrm{NN}$. The second term considers Haldane next-nearest neighbor (NNN) complex hoppings with $\nu_{i j}= \pm 1$, according to the arrows represented in the honeycomb cell in Fig. 2 a. The third term describes the local interaction between localized and itinerant electrons, with $U>0$. The final term contains the chemical potentials for the itinerant and localized electrons, respectively $\mu_{c}$ and $\mu_{f}$. We focus on the case of half-filling for both species - one particle per unit cell - and therefore set $\mu_{c}=\mu_{f}=U / 2$. In what follows, $t=1$ sets the energy scale and $t_{2}=0.1 t$.

Given that the $f$-electron densities $n_{i}^{f}(=0,1)$ can be seen as classical variables, the partition function can be written as

$$
\mathcal{Z}=\sum_{\left\{n_{f}\right\}} \operatorname{Tr}_{c}\left[e^{-\beta \hat{H}\left(\left\{n_{f}\right\}\right)}\right]=\sum_{\left\{n_{f}\right\}} e^{-\beta \mathcal{H}\left(\left\{n_{f}\right\}\right)}
$$

where

$$
\mathcal{H}\left(\left\{n_{f}\right\}\right)=-\frac{U}{2} \sum_{i} n_{i}^{f}-\frac{1}{\beta} \sum_{j} \ln \left[1+e^{-\beta E_{j}\left(\left\{n_{f}\right\}\right)}\right]
$$
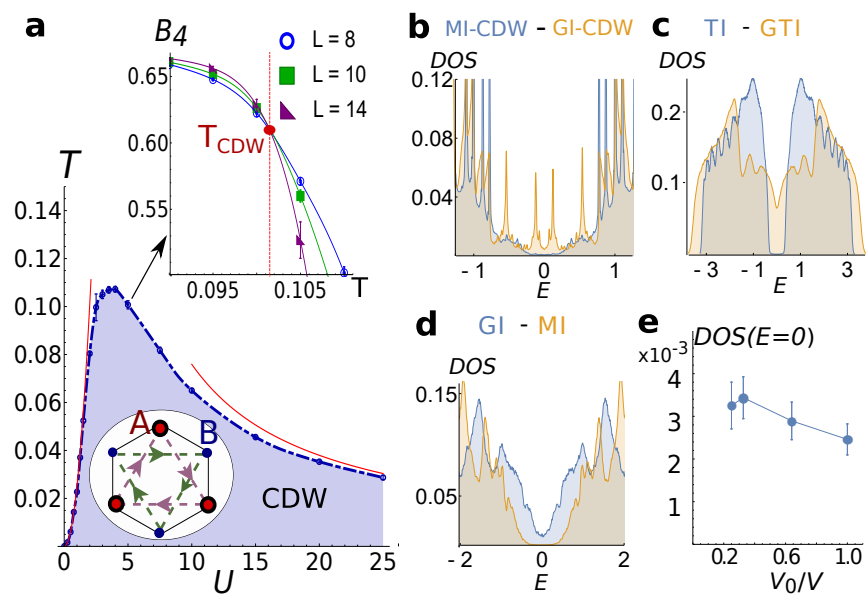

FIG. 2. a, Monte Carlo results of the CDW phase transition along with the small and large $U$ curves obtained with second order perturbation theory [58]. The larger sites in the honeycomb unit cell inside the CDW phase represent occupied sites indicating a checkerboard order; the arrows represent the flow of NNN hoppings. The inset shows an example of the usage of the Binder cumulant method to compute the critical temperature $T_{\mathrm{CDW}}$ that corresponds to the intersection of the obtained curves for different system sizes, for a case where $U=5$. b-d, Density of states for different points in the phase diagram: $\mathrm{MI}-\mathrm{CDW}-(U, T)=(2.5,0.045)$, GICDW-(2.5, 0.085), TI-(1,0.1), GTI- $(2,0.1)$, GI- $(4,0.1)$ and MI- $(5,0.1)$; we use a Lorentzian broadening of width 0.01 . e, Finite-size scaling of the DOS at $E=0$ for the point $(2.5,0.085)$ used in $\mathbf{b}$. $V_{0}$ corresponds to volume of the smallest lattice used (with $L=8$ ); the $\operatorname{DOS}(E=0)$ was computed in an energy window corresponding to $1 \%$ of the full bandwidth for the $L=8$ system. This window was reduced proportionally to the system size for larger systems.

is the effective Hamiltonian obtained after taking the trace over the $c$-electrons' degrees of freedom and is defined in terms of the eigenvalues $E_{j}\left(\left\{n_{f}\right\}\right)$ of $\hat{H}$ obtained for a fixed configuration $\left\{n_{f}\right\}$. In this from, the model is amenable to classical Monte Carlo sampling.

Observables. - For the $f$-electrons, we focus on describing the CDW phase transition, characterized by an order parameter corresponding to the staggered occupation of $f$-electrons on sublattices $A$ and $B, n_{\mathrm{st}}^{f}$. $=$ $n_{A}^{f}-n_{B}^{f}$, where $n_{x}^{f}$ is the density in sublattice $x$. The critical $T$ curve, $T_{\mathrm{CDW}}(U)$, is obtained by fixing $U$ and computing the intersections of the $T$-dependent Binder cumulant, $B_{4}=\left(1-\left\langle n_{\text {st. }}^{f}{ }^{4}\right\rangle / 3\left\langle n_{\text {st. }}^{f}{ }^{2}\right\rangle^{2}\right)$, for different system sizes as shown in the inset of Fig. 2 a. Regarding the $c$-electrons, we investigate the following observables: the Chern number $C$, computed with the method introduced in Ref. [55], specially developed for systems that are not translationally invariant (see discussion for the validity of this approach below); density of states (DOS), obtained with the eigenvalues of the fermionic degrees of freedom; localization of the eigenstates, studied with the inverse participation ratio (IPR) and level spacing statis- 

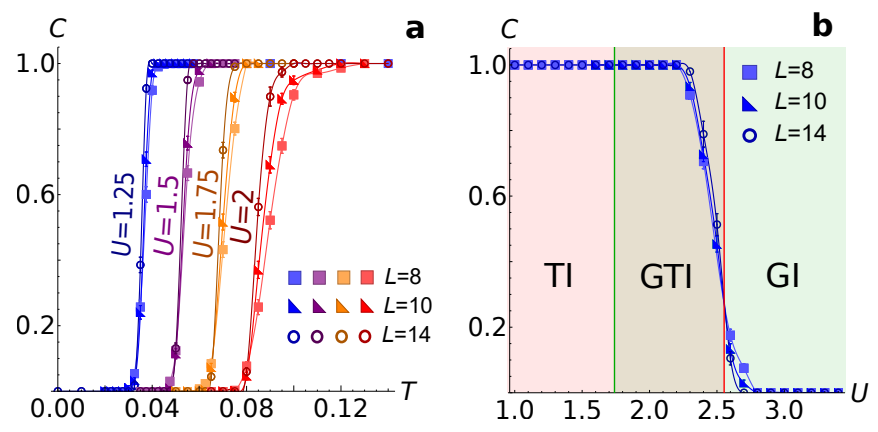

FIG. 3. Chern number computed through averages on Monte Carlo configurations of $f$-electrons for different system sizes, with fixed $U$ (a) and $T$ (b). The crossing points of these curves were used to obtain the topological phase transition curve in Fig. 1.

tics (LSS) methods. In our definition, the IPR is computed for a given energy $E_{\alpha}$ as $\mathcal{I}_{\alpha}=\sum_{i}\left|\phi_{i}^{\alpha}\right|^{4}$, where $\phi_{i}^{\alpha}$ is the amplitude of the eigenvector with energy $E_{\alpha}$ at site $i$. An IPR histogram can then be obtained as a function of the energy by sampling a large number of MC configurations; this quantity scales to zero with the system's volume if we deal with extended states, and to a constant if the states are localized. The LSS also provides a simple way of distinguishing between extended and localized states: for extended states, level repulsion is expected and the spacings between energy levels assume a Wigner distribution with variance $(\sigma /\langle s\rangle)^{2}=0.178$ (for the case of the unitary class to which the HFKM belongs), where $\langle s\rangle$ is the average value of the distribution of level spacings $s$; for localized states, the level spacing distribution acquires a Poisson-like shape with a larger variance [59]. In what follows, we describe the properties of the different phases in Fig. 1.

$(C D W)$. - Below the $T_{\mathrm{CDW}}$ curve, dashed-dotted (blue) line in Figs. 1 and $2 \mathbf{a}$, the $f$-electrons start ordering in a checkerboard-like pattern for which only one of the sublattices is occupied as sketched in the honeycomb cell in Fig. 2 a. To better understand the behavior of the CDW phase transition curve, we perform a mapping to the 2D antiferromagnetic Ising model at small and large $U$ and the phase transition curves can be obtained with a perturbative analysis [58]. These curves were computed up to second order in the perturbation which involves either the terms with $U$ or with the hoppings $t$ and $t_{2}$ and are shown in Fig. 2 a, as full (red) lines. For $U \ll 1$, the $T_{\mathrm{CDW}}(U)$ curve is quadratic whereas at large interactions, it is inversely proportional to $U$ - the agreement with MC is remarkable.

Besides the expected trivial gapped CDW phase (MICDW), as found in Ref. [52] for the FKM, the HFKM additionally hosts a topological insulating phase with charge ordering (TI-CDW) along with a peculiar region of the phase diagram for which the $c$-electron spectrum is gapless inside the CDW phase (GI-CDW). The former had already been noticed in Refs. [33, 60] and contrasts with the results of the spinless fermion Haldane model with NN interactions for which there is no region of coexistence between the CDW and TI phases [2]. Figure. $2 \mathbf{b}$ compares the DOS inside the MI-CDW and GI-CDW phases for $U=2.5$ and $L=16$, for which the transition between gapped and gapless regimes can be clearly seen. To ensure that the GI-CDW phase does not stem from a finite-size effect, we compute the DOS at the Fermi energy $(E=0)$ in an energy window corresponding to $1 \%$ of the total bandwidth and by counting the number of states inside, while decreasing the window's width proportionally to the system size. An example of this scaling is shown in figure Fig. 2 e for a point inside the GI-CDW phase, for which it can be seen that the $\operatorname{DOS}(E=0)$ is stabilized and does not scale to zero.

(TI and GTI). - The TI is a gapped topological phase, i.e., $\operatorname{DOS}(E)=0$ for $|E|<\Delta_{\text {Top }} / 2$ and Chern number $C=1$, with $\Delta_{\text {Top }}$ being the topological gap. At $T=0$, the $f$-electrons only occupy one of the sublattices and therefore act as a staggered potential for the $c$-electrons. This means that the topological insulating phase exists between $U=0$ and $U=6 \sqrt{3} t_{2} \approx 1$, value at which the gap closes and reopens signaling the topological phase transition (TPT) [53]. When we increase $T$, the topological phase still exists and extends to larger $U$. This is expected for the Haldane model with binary disorder - the large $T$ limit, depicted by the vertical lines in the phase diagram (Fig. 1) at $T \gg 1[61]$ - where the topological phase is only destroyed for $U \approx 2.7$, meaning there must be a $T$-driven TPT for $1 \lesssim U \lesssim 2.7$. The corresponding phase transition curve is shown in Fig. 1 as a thin continuous (red) line [62] and some of the Chern number curves used to compile it are shown in Fig. 3. We argue that this $T$-driven topological transition is reminiscent of the TAI phenomenon, for which disorder-induced transitions into topological phases can occur. Although there is no quenched disorder in the system, thermal fluctuations act as to promote an annealed disorder, i.e., the thermal average on the $f$-electron configurations acts as a disordered potential to the $c$-electrons. As a result, the topological phase extends into the gapless region of the phase diagram for higher $T$.

We notice in Fig. 2 c, that the topological gap existing in the TI phase is closed in the GTI phase as one increases the interactions but the Chern number is unchanged $(C=1)$, as can be seen in Fig. 3 b. For the TPT from MI-CDW into the TI phase with increasing $T$, the gap closes and reopens at the phase transition curve. On the other hand, the TPT from the GTI into the GI phase (the GI is further analysed below) is accompanied by the merging of the only two extended states that exist in the spectrum and carry opposite Chern numbers.

Topological phases are robust at finite $T$ provided the thermal fluctuation energy $k_{B} T$ does not exceed the energy separation of the extended states, as all the eigen- 


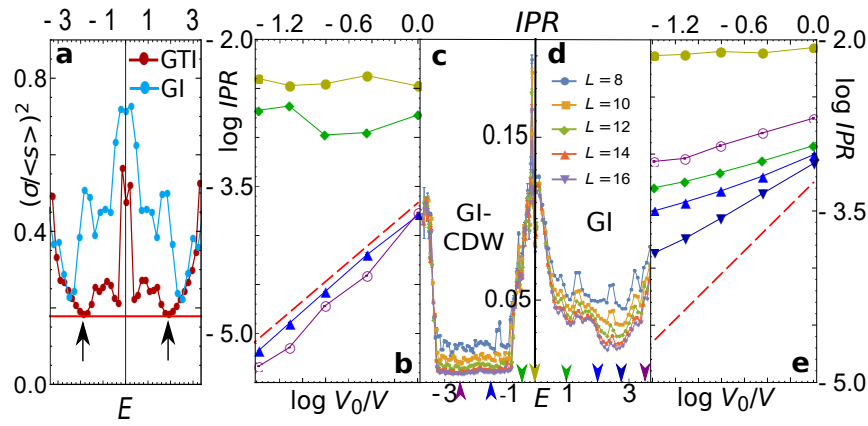

FIG. 4. a, Standard deviations of the LSS distributions obtained for different energies in the GI [GTI] phase for $(U, T)=(2,0.1)[=(3.5,0.2)]$ and $L=14$. The horizontal (red) line corresponds to $\sigma /\langle s\rangle=0.178$ which is the standard deviation of the Wigner distribution associated to extended states. The two extended states existing in the GTI phase are marked with arrows. b (e), Finite size scaling of the IPR with the system volume $V$ for the energies marked with the arrows in $\mathbf{c}$ (d), that shows the IPR for different sizes in the GI-CDW [GI] phase for $(U, T)=(2.5,0.085)[=(3.5,0.2)]$. The IPR shown in c (d) for negative (positive) energies is symmetric in $E$. The red dashed lines shown in $\mathbf{b}, \mathbf{e}$ have a unit slope and indicate the scaling IPR $\sim V^{-1}$. The colors of the arrows that select specific energies in $\mathbf{c}(\mathbf{d})$ match the corresponding scaling curves in $\mathbf{b}(\mathbf{e})$.

states in-between are localized and cannot change the Chern number, similarly to the case of the integer quantum Hall effect. This condition breaks down near the TPT curve of Fig. 1. However, as shown in Fig. 4 a, only slightly away from the TPT line, the extended states already have an energy separation $\Delta E \approx 4 \gg k_{B} T \approx 0.1$, allowing for a $T$-driven TPT into gapped and gapless TIs.

(GI and MI).- Increasing $U$ from the GTI phase leads to an interaction-driven TPT into a trivial gapless insulating phase (GI). If we continue increasing $U$, the $c$-electron spectrum acquires a Mott-like gap (MI). The corresponding DOS within the GI and MI phases is exemplified in Fig. 2 d. This phase transition resembles the one found for the 2D FKM in Ref. [52], for increasing interactions between an Anderson and a Mott insulator.

Gapless insulators. - We report, in Fig. 4 a, the LSS results for the GTI and GI phases. In the GTI phase, the standard deviation of the level spacing distributions, $\sigma /\langle s\rangle$, has the expected value for the Wigner distribution (horizontal line) at the particular energies corresponding to the two extended states that carry opposite Chern numbers. Away from these particular values, and within all the GI phase, $\sigma /\langle s\rangle$ raises above the Wigner distribution prediction signaling the localization of eigenstates. The IPR, for the GI phase, is depicted in Fig. 4 d. Around $E=0$, it is almost unchanged with the system size thus, the corresponding states are undoubtedly localized. However, for larger $|E|$ values, the IPR decreases with the system size. A finite-size analysis is shown in Fig. 4 e, where the unit slope associated with the scaling IPR $\propto V^{-1}$ is depicted by the (red) dashed line. Nonetheless, the slopes at different energies are always smaller than one, suggesting that localization is robust for every energy in the GI phase. These results are compatible with the following scenario: outside the CDW phase, spatial correlations between $f$-electron occupations in GTI and GI phases decay exponentially with a characteristic length $\xi$. For distances larger then $\xi$, the disorder potential felt by the $c$-electrons becomes uncorrelated. These phases smoothly extend to large $T$, for which $\xi \simeq 1$ and where disordered effects become equivalent to those of a binary quenched potential [61].

For the GI-CDW, Figs. $4 \mathbf{b}$ and $4 \mathbf{c}$, a similar analysis suggests that although the eigenstates are localized around $E=0$, there are also regions of extended states. Figure $4 \mathbf{c}$ shows that the IPR becomes smaller with $V$ for $-3 \lesssim E \lesssim-1$ (and $1 \lesssim E \lesssim 3$, not shown) and Fig. $4 \mathbf{b}$ indicates that for two energies in this interval, the IPR indeed scales with $V^{-1}$ for the used system sizes. This is in apparent contradiction with results for $\sigma /\langle s\rangle$ (not shown), where all energies rise above the Wigner distribution prediction indicating that all eigenstates should be localized. These seemingly contradicting facts can be reconciled by noticing that, inside the CDW phase, $\xi$ diverges and the disordered potential experienced by the $c$-electrons becomes long-range correlated. In twodimensions, systems with long-range spatially correlated disorder have been shown to support spectral regions of extended states [63-65], moreover, Wigner distribution predictions are expected not to hold for such kind of disorder.

Figure 1 shows that the GI-CDW phase is created, starting from the $T=0$ gapped CDW, by increasing $T$. Our results show that the gap starts being populated by localized states induced by the thermal fluctuations. Here, again, disorder is correlated and may support extended states for a finite disorder strength. The important question is whether a region of extended states still survives upon entering the GI-CDW phase or if all states are already localized for this value of $T$. Although our results strongly suggest the former, we cannot definitely exclude the latter scenario which will require working with substantially larger systems sizes. If confirmed, the coexistence of spectral regions of extended and localized states would correspond to one of the first examples of a many-body mobility edge in a strong interacting system and may suggest similar phenomena to be present in the case of finite mass-ratio between electronic species.

Summarizing the central result of our work: we introduce the HFKM model allowing to effectively study the interplay of topology and interactions at finite temperatures and provide a complete characterization of the phase diagram. We show the possibility of having a temperature-driven topological phase transition into gapped and gapless topological insulators; finally, we find an insulating charge ordered state with gapless excita- 
tions where spectral regions of extended and localized states seem to coexist due to the long range nature of the interaction-induced disorder potential.

All the ingredients for the experimental realization of the HFKM with ultracold atoms in optical lattices are separately available: there are recent implementations of mass unbalanced fermions [66, 67]; and, the Haldane model has recently been successfully realized [68]. A direct verification of our results should therefore be achievable with state-of-the-art technology.

The M.G., P.R. and E.V.C acknowledge partial support from FCT-Portugal through Grant No. UID/CTM/04540/2013. M.G. and P.R. acknowledge support by FCT-Portugal through the Investigador FCT contract IF/00347/2014. R.M. acknowledges support of the NSFC Grants No. 11674021 and No. 11650110441, and NSAF-U1530401. The computations were performed in the Tianhe-2JK at the Beijing Computational Science Research Center (CSRC).

[1] C. Xu and J. E. Moore, Phys. Rev. B 73, 045322 (2006).

[2] C. N. Varney, K. Sun, M. Rigol, and V. Galitski, Phys. Rev. B 82, 115125 (2010).

[3] S. Rachel and K. Le Hur, Phys. Rev. B 82, 075106 (2010).

[4] Y. Yamaji and M. Imada, Phys. Rev. B 83, 205122 (2011).

[5] D. Zheng, G.-M. Zhang, and C. Wu, Phys. Rev. B 84, 205121 (2011).

[6] S.-L. Yu, X. C. Xie, and J.-X. Li, Phys. Rev. Lett. 107, 010401 (2011).

[7] C. Griset and C. Xu, Phys. Rev. B 85, 045123 (2012).

[8] M. Hohenadler, T. C. Lang, and F. F. Assaad, Phys. Rev. Lett. 106, 100403 (2011).

[9] M. Hohenadler, Z. Y. Meng, T. C. Lang, S. Wessel, A. Muramatsu, and F. F. Assaad, Phys. Rev. B 85, 115132 (2012).

[10] J. Reuther, R. Thomale, and S. Rachel, Phys. Rev. B 86, 155127 (2012).

[11] M. A. N. Araujo, E. V. Castro, and P. D. Sacramento, Phys. Rev. B 87, 085109 (2013).

[12] M. Laubach, J. Reuther, R. Thomale, and S. Rachel, Phys. Rev. B 90, 165136 (2014).

[13] R. S. K. Mong, A. M. Essin, and J. E. Moore, Phys. Rev. B 81, 245209 (2010).

[14] C. Fang, M. J. Gilbert, and B. A. Bernevig, Phys. Rev. B 88, 085406 (2013).

[15] T. Yoshida, R. Peters, S. Fujimoto, and N. Kawakami, Phys. Rev. B 87, 085134 (2013).

[16] S. Miyakoshi and Y. Ohta, Phys. Rev. B 87, 195133 (2013).

[17] S. Raghu, X.-L. Qi, C. Honerkamp, and S.-C. Zhang, Phys. Rev. Lett. 100, 156401 (2008).

[18] J. Wen, A. Rüegg, C.-C. J. Wang, and G. A. Fiete, Phys. Rev. B 82, 075125 (2010).

[19] J. C. Budich, R. Thomale, G. Li, M. Laubach, and S.-C. Zhang, Phys. Rev. B 86, 201407 (2012).

[20] A. Dauphin, M. Müller, and M. A. Martin-Delgado, Phys. Rev. A 86, 053618 (2012).
[21] C. Weeks and M. Franz, Phys. Rev. B 81, 085105 (2010).

[22] L. Wang, X. Dai, and X. C. Xie, Europhys. Lett. 98, 57001 (2012).

[23] A. Rüegg and G. A. Fiete, Phys. Rev. B 84, 201103 (2011).

[24] K.-Y. Yang, W. Zhu, D. Xiao, S. Okamoto, Z. Wang, and Y. Ran, Phys. Rev. B 84, 201104 (2011).

[25] N. A. García-Martínez, A. G. Grushin, T. Neupert, B. Valenzuela, and E. V. Castro, Phys. Rev. B 88, 245123 (2013).

[26] M. Daghofer and M. Hohenadler, Phys. Rev. B 89, 035103 (2014).

[27] J. Motruk, A. G. Grushin, F. de Juan, and F. Pollmann, Phys. Rev. B 92, 085147 (2015).

[28] S. Capponi and A. M. Läuchli, Phys. Rev. B 92, 085146 (2015).

[29] J. C. Budich, B. Trauzettel, and G. Sangiovanni, Phys. Rev. B 87, 235104 (2013).

[30] S. Rachel, arXiv:1804.10656 (and references therein).

[31] Y.-X. Zhu, J. He, C.-L. Zang, Y. Liang, and S.-P. Kou, J. of Phys.: Cond. Matt. 26, 175601 (2014).

[32] Y.-H. Chen, H.-H. Hung, G. Su, G. A. Fiete, and C. S. Ting, Phys. Rev. B 91, 045122 (2015).

[33] D. Zdulski and K. Byczuk, Phys. Rev. B 92, 125102 (2015).

[34] T. Yoshida, S. Fujimoto, and N. Kawakami, Phys. Rev. B 85, 125113 (2012).

[35] A. Rivas, O. Viyuela, and M. A. Martin-Delgado, Phys. Rev. B 88, 155141 (2013).

[36] P. Dziawa, B. J. Kowalski, K. Dybko, R. Buczko, A. Szczerbakow, M. Szot, E. Lusakowska, T. Balasubramanian, B. M. Wojek, M. H. Berntsen, O. Tjernberg, and T. Story, Nat. Mat. 11, 1023 (2012).

[37] A. Altland and M. R. Zirnbauer, Phys. Rev. B 55, 1142 (1997).

[38] F. Evers and A. D. Mirlin, Rev. Mod. Phys. 80, 1355 (2008).

[39] C.-K. K. Chiu, J. C. Y. Teo, A. P. Schnyder, and S. Ryu, Rev. Mod. Phys. 88, 035005 (2016).

[40] M. Onoda and N. Nagaosa, Phys. Rev. Lett. 90, 206601 (2003).

[41] M. Onoda, Y. Avishai, and N. Nagaosa, Phys. Rev. Lett. 98, 076802 (2007).

[42] J. Li, R.-L. Chu, J. K. Jain, and S.-Q. Shen, Phys. Rev. Lett. 102, 136806 (2009).

[43] C. W. Groth, M. Wimmer, A. R. Akhmerov, J. Tworzydło, and C. W. J. Beenakker, Phys. Rev. Lett. 103, 196805 (2009).

[44] J. Song, H. Liu, H. Jiang, Q.-F. Sun, and X. C. Xie, Phys. Rev. B 85, 195125 (2012).

[45] Y. Su, Y. Avishai, and X. R. Wang, Phys. Rev. B 93, 214206 (2016).

[46] C. P. Orth, T. Sekera, C. Bruder, and T. L. Schmidt, Sci. Rep. 6, 24007 (2016), article.

[47] L. M. Falicov and J. C. Kimball, Phys. Rev. Lett. 22, 997 (1969).

[48] U. Brandt and R. Schmidt, Z. Phys. B 63, 45 (1986).

[49] T. Kennedy and E. H. Lieb, Physica A 138, 320 (1986).

[50] M. M. Maśka and K. Czajka, Phys. Rev. B 74, 035109 (2006).

[51] M. Žonda, P. Farkašovský, and H. Čenčariková, Solid State Comm. 149, 1997 (2009).

[52] A. E. Antipov, Y. Javanmard, P. Ribeiro, and S. Kirchner, Phys. Rev. Lett. 117, 146601 (2016). 
[53] F. D. M. Haldane, Phys. Rev. Lett. 61, 2015 (1988).

[54] D. N. Sheng, L. Sheng, Z. Y. Weng, and F. D. M. Haldane, Phys. Rev. B 72, 153307 (2005).

[55] Y.-F. Zhang, Y.-Y. Yang, Y. Ju, L. Sheng, R. Shen, D.N. Sheng, and D.-Y. Xing, Chin. Phys. B 22, 117312 (2013).

[56] E. V. Castro, M. P. López-Sancho, and M. A. H. Vozmediano, Phys. Rev. B 92, 085410 (2015).

[57] E. V. Castro, R. de Gail, M. P. López-Sancho, and M. A. H. Vozmediano, Phys. Rev. B 93, 245414 (2016).

[58] See Supplemental Material for a brief description of the analysis on the perturbative results at large and small interactions.

[59] E. Prodan, J. Phys. A 44, 113001 (2011).

[60] H.-S. Nguyen and M.-T. Tran, Phys. Rev. B 88, 165132 (2013).

[61] M. Gonçalves, P. Ribeiro, and E. V. Castro, arXiv:1807.11247.

[62] We notice that the small $U$ behavior of this curve has been qualitatively predicted with mean-field in Ref. [33].

[63] D. Taras-Semchuk and K. B. Efetov, Phys. Rev. B 64, 115301 (2001).

[64] M. Hilke, Phys. Rev. Lett. 91, 226403 (2003).

[65] F. A. De Moura, M. L. Lyra, F. Domínguez-Adame, and V. A. Malyshev, J. Phys. Cond. Mat. 19 (2007).

[66] G. Jotzu, M. Messer, F. Görg, D. Greif, R. Desbuquois, and T. Esslinger, Phys. Rev. Lett. 115, 073002 (2015).

[67] D. Greif, G. Jotzu, M. Messer, R. Desbuquois, and T. Esslinger, Phys. Rev. Lett. 115, 260401 (2015).

[68] G. Jotzu, M. Messer, R. Desbuquois, M. Lebrat, T. Uehlinger, D. Greif, and T. Esslinger, Nature 515, 237 (2014).

[69] P. Coleman, Introduction to Many-Body Physics (Cambridge University Press, 2015). 


\section{Supplementary Materials: Temperature-driven gapless topological insulators}

\section{PERTURBATIVE ANALYSIS}

By making a perturbative expansion for the effective Hamiltonian $\mathcal{H}\left(\left\{n_{f}\right\}\right)$ defined in Eq. (3) it is possible to study the small and large $U$ regions of the phase diagram. This expansion allows us to write the Hamiltonian of the HFKM in the form of an effective 2D antiferromagnetic Ising model that only depends on the $f$-electrons' degrees of freedom. In this way, we can see the CDW phase transition curves of the effective Ising models as an approximation of the exact phase transition curve of the HFKM on the limits of concern.

We start by defining the Hamiltonian matrix $\boldsymbol{H}$

$$
\boldsymbol{H}=-t \sum_{\langle i, j\rangle}|i\rangle\left\langle j\left|+\mathrm{i} t_{2} \sum_{\langle\langle i, j\rangle\rangle} \nu_{i j}\right| i\right\rangle\left\langle j\left|+\frac{U}{2} \sum_{i} s_{i}\right| i\right\rangle\langle i|,
$$

where we introduced the Ising variables $s_{i}=2 n_{i}^{f}-1=$ \pm 1 . The propagator of $c$-electrons is then simply given by $\boldsymbol{G}=\left(\mathrm{i} \omega_{n}-\boldsymbol{H}\right)^{-1}$. Once the HFKM Hamiltonian is quadratic in the $c$-electron's fields for a given configuration $\left\{n_{f}\right\}$, the formalism of Gaussian path integrals can be employed to write [69]

$$
\mathcal{H}=-\frac{1}{\beta} \operatorname{Tr}_{c} \ln \left(-\boldsymbol{G}^{-1}\right)-\frac{U}{2} \sum_{i} n_{i}^{f},
$$

where the trace is taken over the fermionic degrees of freedom and was extended to incorporate the sum in the Matsubara frequencies. If we separate $\boldsymbol{H}$ in the unperturbed and perturbed terms, respectively $\boldsymbol{H}_{0}$ and $\boldsymbol{H}_{1}$, we can show that

$$
\mathcal{H}=\mathcal{H}_{0}-\frac{U}{2} \sum_{i} n_{i}^{f}+\frac{1}{\beta} \sum_{k=1}^{+\infty} \frac{1}{k !} \operatorname{Tr}\left[\left(\boldsymbol{G}_{0} \boldsymbol{H}_{1}\right)^{k}\right],
$$

where $\boldsymbol{G}_{0}^{-1}=\mathrm{i} \omega_{n}-\boldsymbol{H}_{0}$ and $\mathcal{H}_{0}=-\frac{1}{\beta} \operatorname{Tr} \ln \left(-\boldsymbol{G}_{0}^{-1}\right)$. Equation (6) provides a useful starting point for our per- turbative analysis and can be applied for the small and large $U$ limits. For small $U$, we have that $\boldsymbol{H}_{0}$ and $\boldsymbol{H}_{1}$ contain respectively the hopping and $U$ dependent terms in Eq. (4), while for large $U$, they interchange. For the perturbative analysis, the expansion is made up to second order in $\boldsymbol{H}_{1}$. For small $U$, the effective Ising Hamiltonian $\mathcal{H}_{S U}$ is

$$
\mathcal{H}_{S U}=U^{2} \sum_{i, j} J_{i j}(R) s_{i} s_{j}
$$

where the sum is over pairs of neighbors and

$J_{i j}(R)= \begin{cases}\int_{-\infty}^{+\infty} d \omega \frac{A_{\bigcirc}^{2}}{16 \pi^{3} v^{4}}\left(m^{2}-\omega^{2}\right) K_{0}^{2}\left(\frac{R}{v} \sqrt{\omega^{2}+m^{2}}\right) & , \text { (i) } \\ \int_{-\infty}^{+\infty} d \omega \frac{A_{\bigcirc}^{2}}{16 \pi^{3} v^{4}}\left(m^{2}+\omega^{2}\right) K_{1}^{2}\left(\frac{R}{v} \sqrt{\omega^{2}+m^{2}}\right) & , \text { (ii) }\end{cases}$

In the above expression, $R$ is the absolute distance between sites $i$ and $j, m=3 \sqrt{3} t_{2}$ is the Haldane model's topological gap, $A_{\square}=3 \sqrt{3} a^{2} / 2$ is the area of the honeycomb unit cell (with $a$ being the lattice constant) and $v=3 t / 2$ is the Fermi velocity. Finally, $K_{0}$ and $K_{1}$ are modified Bessel functions of the second kind. Conditions (i) and (ii) correspond respectively to $i$ and $j$ in the same and in different sublattices.

On the other hand, the effective Ising Hamiltonian $\mathcal{H}_{L U}$ for large $U$ is

$$
\mathcal{H}_{L U}=\sum_{\langle i, j\rangle} \frac{t^{2}}{2 U} s_{i} s_{j}+\sum_{\langle\langle i, j\rangle\rangle} \frac{t_{2}^{2}}{2 U} s_{i} s_{j}
$$

Based on the effective Ising models, the critical temperature curves were estimated and are shown in Fig. 2 along with the numerical results. For small $U$, the critical temperature was estimated up to next-NNN and NNN were not considered as they can be neglected for the studied case of $t_{2}=0.1 t$. For large $U$, the second term in $\mathcal{H}_{L U}$ can also be neglected for the case of interest. 